\title{
Prevalence, Pattern and Risk Factors of Severe Acute Malnutrition in Children below Six Months Old in Jos North Central Nigeria
}

\author{
John C, ${ }^{1}$ Adah R, ${ }^{2}$ Caleb R, ${ }^{2}$ Okolo S. \\ ${ }^{1}$ Department of Paediatrics, University of Jos and ${ }^{2}$ Jos University Teaching Hospital, Jos, Nigeria.
}

*Corresponding Author: Dr Collins John. Department of Paediatrics, College of Health Sciences, University of Jos, Nigeria.

Email:cchibunkem@yahoo.com

\begin{abstract}
Severe Acute Malnutrition (SAM) in infants of less than 6 months of age (U6m) is a serious public health concern globally, especially with declined rates of exclusive breastfeeding. With challenges in the use of the standard anthropometric parameters and higher mortality in these infants than in older children, it is pertinent to document the burden, pattern and risk factors for SAM in U6m. This study aimed to determine the prevalence of SAM in U6m, the pattern and the determinants of malnutrition using weight-for-length (WFL) Z score and mid-upper arm circumference (MUAC), and also to determine the usability of MUAC and WFL in detection of SAM in U6m. Infants six weeks to $<6$ months were recruited. Socio-demographic and nutritional data were collected using researcher administered questionnaire. WFL z scores $<-3$ and MUAC $<11.5 \mathrm{~cm}$ were used to define SAM. Of the 233 infants aged $<6$ months, mean age was $90.0 \pm 38.3$ days. Females accounted for $52.8 \%$. Nineteen, $(8.5 \%)$, were born with low birth weight and $11.7 \%$ were preterm deliveries. Males had higher mean length for age and weight for age than females but MUAC showed no significant difference. The prevalence of SAM was $2.6 \%$ by MUAC and WFL parameters but MUAC identified more SAM subjects in those $<3$ months while WFL identified more in older children. Concordance between MUAC and WFL was poor. Both MUAC and WFL showed more female children than males with MAM. Lower social economic status was significantly associated with SAM while birth weight, birth order, maternal nutritional status and time of first feed did not affect prevalence of SAM in any of the parameters. It is recommended that both WFL and MUAC be employed in the screening of acute malnutrition in this U6m.
\end{abstract}

Keywords: Acute malnutrition,Under 6months, MUAC and WFL.

\section{INTRODUCTION}

$\mathrm{C}$ hildhood wasting is a serious public health concern worldwide. ${ }^{1}$ High rate of malnutrition pose significant public health and development challenges for the country. With declined and varying rates of exclusive breastfeeding among several population groups ${ }^{2}$, severe acute malnutrition is increasingly becoming a recognized entity in infants less than 6 months of age (U6m). It is also said to be associated with higher mortality in these infants than in older infants and children. ${ }^{3}$ Kerac et al reported that globally, some 4.7 million infants less than 6 months of age are moderately wasted and 3.8 million are severely wasted, and these set of persons have been overlooked by clinicians, nutritionists, and policy makers. ${ }^{4}$ The diagnosis of SAM in U6m utilizes weight for length (WFL) $<-3$ standard deviation ${ }^{5}$, this as seen in 
children 6-59 months is not a good predictor of mortality. ${ }^{6}$ The use of MUAC in U6m has not been validated but has shown some advantages such as its simplicity and better interobserver reliability than WFL. ${ }^{7}$ Both methods have been reported to identify different subsets of SAM patients with poor concordance. ${ }^{8,9}$ Thus WFL does not give a clear picture of the burden of SAM in U6m hence the need to seek more reliable clinical and field parameters for identification of SAM in U6m as opined by many authors. ${ }^{69,10}$

Little is known about the burden of severe acute malnutrition in children less than 6months (U6m) in Nigeria using the defined parameters. There is also dearth of information on risk factors that determine the prevalence of malnutrition in this population group.

The paucity of data therefore hinders appropriate policy development and implementation. This study was therefore designed to determine the prevalence of SAM among children less than 6months of age, the pattern of malnutrition using various anthropometric indices and the risk factors of malnutrition in this population. It also intends to determine if MUAC can be used in the diagnosis of SAM in this subset of children.

\section{MATERIALS \& METHODS}

\section{Study design}

This was a cross sectional study of children six weeks to $<6$ months attended to at the Jos University Teaching Hospital paediatric outpatient department and immunization clinic. All infants age less than 6months seen at the clinic, whose mothers gave consent, were consecutively recruited until the sample size was met.

\section{Ethical Approval}

Ethical Approval for the study was received from the institutional review board of the Jos University teaching hospital. Consent was also obtained from mothers of babies recruited for the study.

\section{Data collection}

Infant feeding practices from birth till date was assessed using a semi-structured researcher administered questionnaire. Anthropometric data which included weight, length, head circumference and mid-upper arm circumference (MUAC) was measured using a digital weighing scale for weight, and infantometer for length, a non-stretchable tape for head circumference and MUAC tape for MUAC. Z scores of length, weight and Weight for Length were derived using WHO Z score charts. All measurements were done using standard methods by trained research assistants.

\section{Sample size determination}

The sample size for the study was computed using EPI info Statcalc at a confidence interval of $95 \%$ and a confidence limit of $5 \%$. Using a prevalence of $13.3 \%$ by Thurstan $\mathrm{S}^{11}$, the calculated minimum sample size was 177 .

\section{RESULTS}

In all, 233 infants aged 6weeks to less than 6months were recruited over a six months period from November 2018 to April 2019. The mean age was 90.2 days \pm 38 .8 days (3.01 \pm 1.3 months). Females were 110 (47.2\%) while males were $123(52.8 \%)$, Female: Male ratio was 0.9:1. Among the subjects, $19(8.5 \%)$ were born with birth weight less than $2.5 \mathrm{~kg}$ and $3.2 \%$ were born with birth weight $4.0 \mathrm{~kg}$ and above. This is shown in Table 1.

\section{Nutritional status}

Males were significantly taller (HAZ) and heavier (WAZ) than the females but there were no significant difference in

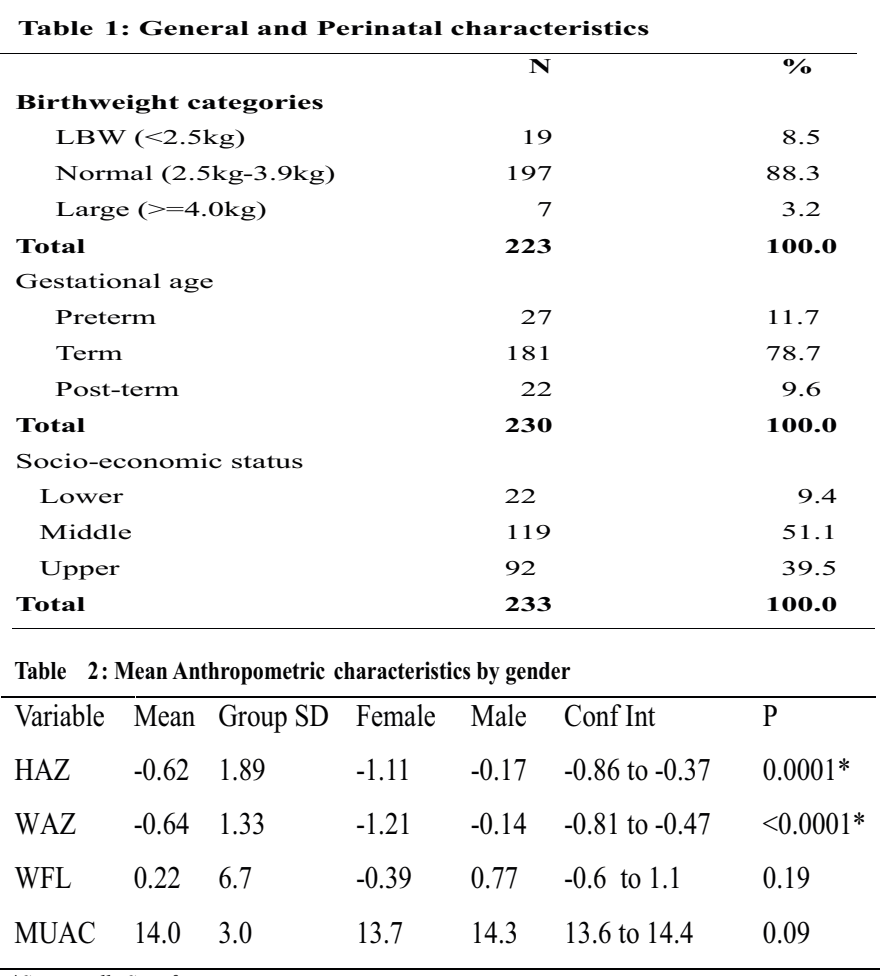

*Statistically Significant 
WFL and mean MUAC measurements between the genders. This is as shown in Table 2. MUAC showed no significant gender difference although mean MUAC was more in males than females. The prevalence of SAM by WFL was $2.6 \%$, females had a higher prevalence of SAM, MAM and consequently GAM but not significantly different from males $(\mathrm{p}=0.4)$. When MUAC was used, prevalence of SAM was also $2.6 \%$, but a higher prevalence of MAM- $10.3 \%$ than WFL $(6.9 \%)$ identified, while SAM was higher in males $(3.3 \%$ vs $1.8 \%$ ) with MUAC than with WFL. Generally more female infants were stunted and undernourished than male using HAZ and WAZ (Table 3).

\section{Risk Factors}

By age group, infants more than three months had more SAM patients identified by WFL while MUAC identified more SAM cases in children less than 3 months. No child above three months was identified as MAM using MUAC. This is shown in table 4.

Birth order, birth-weight, time of first feed, and maternal nutrition had no significant impact on prevalence of SAM either by WFL or MUAC. WFL demonstrated significant difference in prevalence of acute malnutrition among the different socio-economic strata. MUAC showed no significant difference

Table 3: Nutritional status by Gender

\begin{tabular}{|c|c|c|c|c|}
\hline Variable & Group & Female & Male & $\mathrm{P}$ \\
\hline WFL & $\mathrm{n}(\%)$ & $\mathrm{n}(\%)$ & $\mathrm{n}(\%)$ & \\
\hline SAM $(<-3)$ & $6(2.6)$ & $3(2.7)$ & $3(2.4)$ & 0.4 \\
\hline $\operatorname{MAM}(<-2)$ & $16(6.9)$ & $10(9.1)$ & $6(4.9)$ & \\
\hline Normal $(>=-2)$ & $211(90.6)$ & $97(88.2)$ & $114(92.7)$ & \\
\hline \multicolumn{5}{|l|}{ MUAC } \\
\hline $\mathrm{SAM}<11.5 \mathrm{~cm}$ & $6(2.6)$ & $2(1.8)$ & $4(3.3)$ & 0.43 \\
\hline MAM 11.5-12.4 & $24(10.3)$ & $14(12.7)$ & $10(8.1)$ & \\
\hline Normal 12.5+ & $203(87.1)$ & $94(85.5)$ & $109(88.6)$ & \\
\hline \multicolumn{5}{|l|}{ HAZ } \\
\hline Severe $<-3$ & $15(6.4)$ & $10(9.1)$ & $5(4.1)$ & $0.013 *$ \\
\hline Stunting $<-2$ & $21(9.0)$ & $15(13.6)$ & $6(4.9)$ & \\
\hline Normal $>=-2$ & $197(84.6)$ & $85(77.3)$ & $112(91.0)$ & \\
\hline \multicolumn{5}{|l|}{ WAZ } \\
\hline Severe $<-3$ & $13(5.6)$ & $9(8.2)$ & $4(3.3)$ & $0.000^{*}$ \\
\hline Moderate $<-2$ & $18(7.7)$ & $16(14.5)$ & $2(1.6)$ & \\
\hline Normal $>=-2$ & $202(86.7)$ & $85(77.3)$ & $117(95.1)$ & \\
\hline
\end{tabular}

* statistically significant differences between the genders
Table 4 : Nutritional status by Age groups

\begin{tabular}{llcl}
\hline Variable & $0-3$ months & $>3$ months & P value \\
WFL & $\mathrm{n}(\%)$ & $\mathrm{n}(\%)$ & \\
SAM & $1(0.9)$ & $5(4.3)$ & 0.1 (Fisher's) \\
MAM & $11(9.4)$ & $5(4.3)$ & \\
Normal & $105(89.7)$ & $106(91.4)$ & \\
MUAC & & & \\
SAM & $5(4.3)$ & $1(0.9)$ & $<0.001 *$ (Fisher's) \\
MAM & $24(20.5)$ & $0(0.0)$ & \\
Normal & $88(75.2)$ & $115(99.1)$ & \\
\hline
\end{tabular}

*statistically significant

Table 5: Nutritional status by SES

\begin{tabular}{lllrl}
\hline Variable & Lower & \multicolumn{1}{l}{ Middle } & \multicolumn{1}{c}{ Upper } & P value \\
WFL & $\mathrm{n}(\%)$ & $\mathrm{n}(\%)$ & $\mathrm{n}(\%)$ & \\
SAM & $1(4.5)$ & $3(2.5)$ & $2(2.2)$ & $0.042^{*}$ \\
MAM & $4(18.2)$ & $10(8.4)$ & $2(2.2)$ & \\
Normal & $17(77.3)$ & $106(89.1)$ & $88(95.6)$ & \\
Total & $\mathbf{2 2 ( 9 . 4 )}$ & $\mathbf{1 1 9}(\mathbf{5 1 . 1})$ & $\mathbf{9 2}(\mathbf{3 9 . 5 )}$ & \\
MUAC & & & & \\
SAM & $2(33.3)$ & $4(66.7)$ & $0(0.0)$ & 0.112 \\
MAM & $1(4.2)$ & $14(58.3)$ & $9(37.5)$ & \\
Normal & $19(9.4)$ & $101(49.7)$ & $83(40.9)$ & \\
Total & $\mathbf{2 2 ( 9 . 4 )}$ & $\mathbf{1 1 9}(\mathbf{5 1 . 1})$ & $\mathbf{9 2}(\mathbf{3 9 . 5})$ & \\
\hline Statistically & signifint & &
\end{tabular}

* Statistically significant

Table 6: Comparison of Nutritional status by MUAC and WFL

\begin{tabular}{|c|c|c|c|c|}
\hline MUAC & WFL $<-3$ & WFL $<-2$ & $\mathbf{N}$ & Total \\
\hline & n $\quad(\%)$ & n $(\%)$ & n $(\%)$ & n $\quad(\%)$ \\
\hline SAM & $1(16.7)$ & $2(12.5)$ & $(1.4)$ & $6(2.6)$ \\
\hline MAM & $0(0.00)$ & $4 \quad(25.0)$ & $(9.5)$ & $24(10.3)$ \\
\hline Normal & $5(83.3)$ & $10(62.5)$ & 188 (89.1) & $203(87.1)$ \\
\hline Total & $6(100.0)$ & $16(100.0)$ & $211(100.0)$ & $233(100.0)$ \\
\hline
\end{tabular}

$\mathrm{P}=0.005$, statistically significant

\section{DISCUSSION}

This study demonstrates the prevalence and pattern of acute malnutrition in children below six months (U6m). The prevalence of wasting (WFL $<-2)$ among the population was found to be $9.5 \%$ while global acute malnutrition (GAM) using MUAC was $12.9 \%$.

The prevalence of severe acute malnutrition in the study was $2.6 \%$ using WFL. This falls within the global range 
of SAM as reported by Kerac M et al. ${ }^{10}$ and also similar to finding by Prost et al. $^{12}$ among Malawian children 1-4months of age but is less than $6.3 \%$ reported in Kenya from a retrospective study $^{13}$ as well as the $14.8 \%$ reported by Choudhary et al $^{14}$ in the Indian National Family Health Survey Four. Murphy et al. ${ }^{15}$ reported a $7.5 \%$ prevalence utilizing MUAC of $<11.0 \mathrm{~cm}$ in assessment of wasting in infants under 6 months among a refugee population in South Sudan. This parameter, although assessed in this study, has not been validated for use in $\mathrm{U} 6 \mathrm{~m}$ but we found a lower prevalence of $2.6 \%$ using a MUAC cut-off point of $<11.5 \mathrm{~cm}$.

Although not validated in U6m, Mwangome et al. ${ }^{6}$ in a retrospective study showed that infants aged 6 to 14 weeks with MUAC $<11.5 \mathrm{~cm}$ had a fourfold greater risk of dying than those with $M U A C \geq 13.0 \mathrm{~cm}$, but the $11.5 \mathrm{~cm}$ cut-off identified about one fourth of the infants in the sample and hence lacks specificity. In our study, prevalence of SAM using MUAC was same as the prevalence using WFL but no significant correlation between the two parameters. This is similar to our earlier reports ${ }^{8}$ and those of Laillou et al. ${ }^{9}$ that MUAC and WFL identifies different subset of children with malnutrition. It will thus be beneficial to apply both parameters in identification of U6m with acute malnutrition. This is especially important as reports ${ }^{16,17}$ indicate, that MUAC is a better predictor of mortality in SAM than WHZ among younger children and. In this study, we found that the number of children with SAM and MAM using MUAC was significantly more in infants less than 3 months of age. Thus the application of both parameters is of special importance in U6m.

The burden of MAM in this study using WFL was high at $6.9 \%$. There is to the best of the author's knowledge, there is no report on the burden of MAM or SAM in Nigeria among infants less than six months. MAM can proceed to SAM if unidentified and properly managed especially in U6m who maybe receiving inappropriate feeds at this age. This study demonstrates mean differences in MUAC, length for age and weight for age among the sexes but not a significant difference in prevalence of severe acute malnutrition among the sexes. However, prevalence of moderate acute malnutrition using both MUAC and WFL parameters showed more females with moderate malnutrition than the males. MUAC identified more of the MAM than WHZ among the female sex. This is similar to findings by Wieringa et al. ${ }^{18}$ that MUAC identifies more younger female children with malnutrition although in our study WFL did not show more male predominance as in the aforementioned study. ${ }^{18}$

We demonstrate the impact of socio-economic status (SES) on the burden of malnutrition. The lower the SES, the higher the burden of acute malnutrition using WFL parameters, but not with MUAC. The effect of SES on malnutrition is in keeping with findings from Ghana by Novignon $\mathrm{J}$ et $a l^{19}$ that there exists a pro-poor inequality in child malnutrition measured by stunting and wasting.

\section{CONCLUSION}

In all, the prevalence of SAM in children less than six months is low in our setting but WFL and MUAC identifies different subset with little concordance. Pattern of malnutrition shows higher burden in children less than three months, those from lower SES and higher burden of MAM in females using MUAC.

\section{Consent for publication}

All authors in this study have given their consent for publication

\section{Availability of data and material}

All data for this work is available on request

\section{Competing interests}

None

\section{Funding}

None

\section{Authors' contributions}

All authors made significant contribution to design, data collection, manuscript writing, review and editing.

\section{REFERENCES}

1. Manary MJ, Sandige HL. Management of acute moderate and severe childhood malnutrition. BMJ 2008;337:a2180

2. Jones, KM., Power, ML., Queenan, JT., \& Schulkin, J. Racial and ethnic disparities in breastfeeding. Breastfeeding medicine, 2015; 10(4), 186-196. doi: $10.1089 / \mathrm{bfm} .2014 .0152$

3. Grijalva-Eternod, CS., Kerac, M., McGrath, M., Wilkinson, C., Hirsch, JC., Delchevalerie, P., \& Seal, A. J. Admission profile and discharge outcomes for infants 
aged less than 6 months admitted to inpatient therapeutic care in 10 countries. A secondary data analysis. Maternal and Child Nutrition, $2017 ; 13$ (3). https://doi.org/10.1111/mcn.12345

4. Kerac M, Mwangome M, McGrath M, et al. Management of acute malnutrition in infants aged under 6 months (MAMI): current issues and future directions in policy and research. Food Nutr Bull. 2015;36:S30-S34

5. WHO. Guideline: updates on the management of severe acute malnutrition in infants and children. WHO; 2013. A v a i 1 a b 1 e fro m : http://apps.who.int/iris/bitstream/10665/95584/1/978924 1506328 eng.pdf.

6. Mwangome MK, Fegan G, Fulford T, Prentice AM, Berkley JA. Midupper arm circumference at age of routine infant vaccination to identify infants at elevated risk of death: a retrospective cohort study in the Gambia. Bull World Health Organ 2012;90: 887-94.

7. Mwangome MK, Fegan G, Mbunya R, Prentice AM, Berkley JA. Reliability and accuracy of anthropometry performed by community health workers among infants under 6 month in rural Kenya. Trop Med Int Health 2012; 17: 622-9 doi: 10.1111/j.1365-3156.2012.02959.

8. John C, Diala U, Adah R, Lar L, Envuladu EA, Adedeji I, et al. Survival and nutritional status of children with severe acute malnutrition, six months post-discharge from outpatient treatment in Jigawa state, Nigeria. PLOS ONE, $2018 ; \quad 13(6)$ : $\quad$ e 0196971 . https://doi.org/10.1371/journal.pone.0196971

9. Laillou A., Prak S., de Groot R., Whitney S., Conkle J., Horton L., Un S.O., Dijkhuizen M.A., Wieringa F.T. Optimal screening of children with acute malnutrition requires a change in current $\mathrm{WHO}$ guidelines as MUAC and WHZ identify different patient groups. PLOS ONE. 2014;9:e101159. doi: 10.1371/journal.pone.0101159

10. Kerac M, Blencowe H, Grijalva-Eternod C, McGrath M, Shoham J, Cole TJ, Seal A. Prevalence of wasting among under 6-month-old infants in developing countries and implications of new case definitions using WHO growth standards: a secondary data analysis. Arch Dis Child 2011;96:1008-13.

11. Thurstan S. Risk factors associated with severe acute malnutrition in infants under six months in India: a cross sectional analysis. Field Exchange 49, March 2015. p34. www.ennonline.net/fex/49/india

12. Prost M-A., Jahn A., Floyd S., Mvula H., Mwaiyeghele E.,
Mwinuka V., et al. (2008) Implication of New WHO Growth Standards on Identification of Risk Factors and Estimated Prevalence of Malnutrition in Rural Malawian I n f a n ts. P L o S ONE 3( 7 ): e 2684 . https://doi.org/10.1371/journal.pone.0002684

13. Gachau S., Irimu G., Ayieko P., Akech S., Agweyu A., English M; Clinical Information Network Author Group. Prevalence, outcome and quality of care among children hospitalized with severe acute malnutrition in Kenyan hospitals: A multi-site observational study. PLoS One. 2018 May $17 ; 13$ (5): e 0197607 . do i : 10.1371/journal.pone.0197607.

14. Choudhary T.S., Srivastava A., Chowdhury R., et al. Severe wasting among Indian infants $<6$ months: Findings from the National Family Health Survey 4. Matern Child Nutr. 2019; 15 :e 12866. https://doi.org/10.1111/mcn.12866

15. Murphy MT., Abebe K., O'Mahony S, Barthorp H., and Andert C. Management of acute malnutrition in infants less than six months in a South Sudanese refugee population in Ethiopia. Field Exchange 55, July 2017. p70.www.ennonline.net/fex $/ 55 /$ mamisudanrefugees

16. Rasmussen J., Andersen A., Fisker A.B., Ravn H., Sodemann M., Rodrigues A., et al. Mid-upper-armcircumference and mid-upper-arm circumference $\mathrm{z}$ score: the best predictor of mortality? Eur J Clin Nutr. 2012;66:998-1003 doi: 10.1038/ejen.2012.95

17. Sachdeva S., Dewan P., Shah D., Malhotra R.K., Gupta P. Mid-upper arm circumference v. Weight-for-height Zscore for predicting mortality in hospitalized children under 5 years of age. Pub Health Nutr. 2016; 19:2513-2520

18. Wieringa, FT., Gauthier, L., Greffeuille, V., Som, SV., Dijkhuizen, M A., Laillou, A. Poirot, E. Identification of Acute Malnutrition in Children in Cambodia Requires Both Mid Upper Arm Circumference and Weight-ForHeight to Offset Gender Bias of Each Indicator. Nutrients, 2018; 10(6), 786. doi:10.3390/nu10060786

19. Novignon, J., Aboagye, E., Agyemang, OS., \& Aryeetey, G. Socioeconomic-related inequalities in child malnutrition: evidence from the Ghana multiple indicator cluster survey. Health economics review, 2015; 5(1), 34. doi:10.1186/s13561-015-0072-4 